\title{
In vitro evaluation of novel antiviral activities of 60 medicinal plants extracts against hepatitis B virus
}

\author{
AHMED HASSAN ARBAB ${ }^{1,2}$, MOHAMMAD KHALID PARVEZ $^{1}$, \\ MOHAMMED SALEM AL-DOSARI ${ }^{1}$ and ADNAN JATHLAN AL-REHAILY ${ }^{1}$ \\ ${ }^{1}$ Department of Pharmacognosy, College of Pharmacy, King Saud University, \\ Riyadh 11451, Kingdom of Saudi Arabia; ${ }^{2}$ Department of Pharmacognosy, College of Pharmacy, \\ Omdurman Islamic University, Omdurman 14415, Republic of the Sudan
}

Received April 5, 2016; Accepted March 3, 2017

DOI: $10.3892 /$ etm.2017.4530

\begin{abstract}
Currently, $>35$ Saudi Arabian medicinal plants are traditionally used for various liver disorders without a scientific rationale. This is the first experimental evaluation of the anti-hepatitis B virus (HBV) potential of the total ethanolic and sequential organic extracts of 60 candidate medicinal plants. The extracts were tested for toxicity on HepG2.2.15 cells and cytotoxicity concentration $\left(\mathrm{CC}_{50}\right)$ values were determined. The extracts were further investigated on HepG2.2.15 cells for anti-HBV activities by analyzing the inhibition of HBsAg and $\mathrm{HBeAg}$ production in the culture supernatants, and their half maximal inhibitory concentration $\left(\mathrm{IC}_{50}\right)$ and therapeutic index (TI) values were determined. Of the screened plants, Guiera senegalensis (dichloromethane extract, $\mathrm{IC}_{50}=10.65$ ), Pulicaria crispa (ethyl acetate extract, $\mathrm{IC}_{50}=14.45$ ), Coccinea grandis (total ethanol extract, $\mathrm{IC}_{50}=31.57$ ), Fumaria parviflora (hexane extract, $\mathrm{IC}_{50}=35.44$ ), Capparis decidua (aqueous extract, $\mathrm{IC}_{50}=66.82$ ), Corallocarpus epigeus (total ethanol extract, $\mathrm{IC}_{50}=71.9$ ), Indigofera caerulea (methanol extract, $\mathrm{IC}_{50}=73.21$ ), Abutilon figarianum (dichloromethane extract, $\mathrm{IC}_{50}=99.76$ ) and Acacia oerfota (total ethanol extract, $\left.\mathrm{IC}_{50}=101.46\right)$ demonstrated novel anti-HBV activities in a time- and dose-dependent manner. Further qualitative phytochemical analysis of the active extracts revealed the presence of alkaloids, tannins, flavonoids and saponins, which are attributed to antiviral efficacies. In conclusion, $P$. crispa, G. senegalensis and F. parviflora had the most promising anti-HBV potentials, including those of $C$. decidua, C. epigeus, A. figarianum, A. oerfota and I. caerulea with
\end{abstract}

Correspondence to: Dr Mohammad Khalid Parvez, Department of Pharmacognosy, College of Pharmacy, King Saud University, PO Box 2457, Riyadh 11451, Kingdom of Saudi Arabia

E-mail: khalid_parvez@yahoo.com; mohkhalid@ksu.edu.sa

Key words: hepatitis B virus, anti-hepatitis B virus plants, Abutilon figarianum, Acacia oerfota, Capparis deciduas, Coccinea grandis, Corallocarpus epigeus, Fumaria parviflora, Guiera senegalensis, Indigofera coerulea, Pulicaria crispa marked activities. However, a detailed phytochemical study of these extracts is essential to isolate the active principle(s) responsible for their novel anti-HBV potential.

\section{Introduction}

Hepatitis B virus (HBV) is responsible for $\sim 2$ billion cases of liver infection worldwide, including $~ 40 \%$ of chronic carriers at the risk of developing fulminant hepatitis, cirrhosis and hepatocellular carcinoma $(1,2)$. In total, $>50 \%$ of the worldwide population lives in areas where HBV infection is highly endemic and $>75 \%$ of this occurs in Asia, Africa and the Middle East, including the Kingdom of Saudi Arabia $(1,3,4)$. Unfortunately, despite their high efficacies, all currently approved drugs against chronic hepatitis B have their own limitations. While long-term treatment with nucleot(s)ide analogues lead to the emergence of drug-resistance, chemotherapy with interferon- $\alpha$ is associated with a high incidence of adverse effects $(3,4)$. Despite the global success of HBV vaccination programs, vaccine-escape mutants of the virus present another bottleneck in the preventive measures $(1,2)$. In addition, the marketed anti-HBV agents are too expensive for the majority of developing countries. Therefore, there is an urgent need to search for novel anti-HBV agents with greater efficacy and safety.

Currently, there is an ongoing effort to identify anti-HBV products from a variety of plants and natural sources. Notably, it has been estimated that $\sim 80 \%$ of Chinese patients with chronic hepatitis B (CHB) rely on traditional herbal remedies. Compared with the treatment of conventional drugs, such as interferons or lamivudine, a meta-analysis of clinical trials suggested that herbal preparations from Phyllanthus urinaria and Scutellaria baicalensis alone may have equivalent or better effects than lamivudine in the treatment of CHB (5). Additionally, many phytocompounds of different chemical classes have been reported to have promising anti-HBV activities (6-11).

Out of $>1,000$ species of medicinal plants documented in Saudi Arabia, at least 35 plants are used in Saudi folk medicine for the treatment of liver disorders $(12,13)$. However, this wealth of herbal medicine has not been subjected to sustained scientific evaluations of their anti-HBV potentials to date. 
Therefore, the primary aim of the present study was to investigate the in vitro anti-HBV activities of 60 candidate plants and to perform a qualitative phytochemical analysis in order to identify the major secondary metabolites.

\section{Materials and methods}

Selection criteria. Candidate plants in the present study were selected on the basis of following one or more criteria: i) Claimed efficacies in treating various liver diseases in folk or traditional medicine; ii) reported in vitro or in vivo hepatoprotective potentials; iii) published antiviral activities against genetically-close human viruses, such as human immunodeficiency virus (HIV) and herpes simplex virus (HSV); and iv) taxonomically related to plants known for their antiviral activities.

Plant materials. A total of 60 medicinal plants were collected from different regions of the Kingdom of Saudi Arabia $(n=57)$ as well as Sudan $(n=3)$ (Table I). Plants were identified by an experienced plant taxonomist at the College of Pharmacy, King Saud University, (Riyadh, Saudi Arabia) and voucher specimens (Table I) were deposited at the college herbarium.

Preparation of the plants extracts. Dried plant materials were ground to a coarse powder using a mortar and pestle, extracted with $80 \%$ ethanol for 3 days followed by filtering with Whatman Grade 1 paper and were concentrated using a rotary evaporator (Buchi Labortechnik AG, Flawil, Switzerland) under reduced pressure at $4^{\circ} \mathrm{C}$. Plants extracts showing anti-HBV activity were further extracted sequentially with different organic solvents of increasing polarity: Hexane, ethyl acetate, dichloromethane, methanol (all from Merck, Darmstadt, Germany), including the aqueous phase. Briefly, $100 \mathrm{~g}$ of each plant powder was soaked in a suitable volume of hexane with intermittent shaking for $24 \mathrm{~h}$, and filtered using Whatman Grade 1 paper. Each of the residues were further extracted twice with fresh solvent, and the filtrates were pooled together. The residue was air-dried followed by sequential extractions with dichloromethane, ethyl acetate, methanol and double-distilled water similar to the procedure performed for hexane. Finally, solvents were removed under reduced pressure at $4^{\circ} \mathrm{C}$ using a rotary evaporator (Buchi Labortechnik AG). Following complete drying, the yield percentage of each extract was calculated (Table II). For biological screening, each extract was dissolved in dimethyl sulfoxide (DMSO; Sigma-Aldrich, Merck KGaA), and the stocks (100 mg/ml) were stored at $-20^{\circ} \mathrm{C}$ until subsequent use.

Cell culture and drug. The HBV reporter cell line (HepG2.2.15) was a generous gift from Dr Shahid Jameel (International Center for Genetic Engineering \& Biotechnology, New Delhi, India). HepG2.2.15 cells were grown in RPMI-1640 medium (Gibco; Thermo Fisher Scientific Inc., Waltham, MA, USA) supplemented with $10 \%$ heat-inactivated bovine serum (Gibco; Thermo Fisher Scientific, Inc.), 1X penicillin-streptomycin, and $1 \mathrm{X}$ sodium pyruvate streptomycin (HyClone; GE Healthcare Life Sciences, Logan, UT, USA) at $37^{\circ} \mathrm{C}$ in a humidified chamber $\left(5 \% \mathrm{CO}_{2}\right)$. Lamivudine (Sigma-Aldrich; Merck
$\mathrm{KGaA}$ ), the approved anti-HBV nucleoside analog-based drug was used as a standard.

Cytotoxicity assessment of the plants extracts. Cytotoxicity of extracts was tested on HepG2.2.15 cells using a 3-(4,5dimethylthiazol-2-yl)-2,5-diphenyltetrazolium bromide (MTT) cell proliferation assay kit (Tervigen, Gaithersburg, MD, USA) to determine the extract concentrations (doses) that did not affect cell viability, and were used in subsequent assays. MTT assay is based on the metabolic reduction of soluble MTT by mitochondrial enzyme activity of viable cells, into an insoluble colored formazan product which can be measured optically (14). Cells were seeded $\left(0.5 \times 10^{5}\right.$ cells $/ 100 \mu \mathrm{l} /$ well $)$ in flat-bottom 96 -well tissue culture plates (Corning Inc., Corning, NY, USA). Following $24 \mathrm{~h}$ of incubation, the cells were treated (in triplicate) with various concentrations of test samples $(0,6.25,12.5,25,50$ and $100 \mu \mathrm{g} / \mathrm{ml})$ prepared in culture media, and incubated at $37^{\circ} \mathrm{C}$ for $48 \mathrm{~h}$. The final concentration of DMSO never exceeded $0.1 \%$ in any of the assays and therefore, had no signs of toxicity. Blank (only media) and untreated/negative (0.1\% DMSO in media) controls were also included. Cells were treated with MTT reagent (10 $\mu \mathrm{l} /$ well) and further incubated at $37^{\circ} \mathrm{C}$ for 3-4 h. Upon appearance of a purple color, the detergent solution $(100 \mu \mathrm{l})$ from the kit was added to each well and further incubated at $37^{\circ} \mathrm{C}$ for $1 \mathrm{~h}$. Optical density (OD) was recorded at $570 \mathrm{~nm}$ using a microplate reader (ELx800; BioTek Instruments, Inc., Winooski, VT, USA). Non-linear regression analysis was performed using Excel software (Microsoft Corp., Redmond, WA, USA) to determine the concentration that resulted in a $50 \%$ cytotoxicity concentration $\left(\mathrm{CC}_{50}\right)$ using the following equation:

$$
\text { Survival fraction }=\frac{O D[\mathrm{~s}]-O D[b]}{O D[c]-O D[b]}
$$

Where $\mathrm{OD}(\mathrm{s}), \mathrm{OD}(\mathrm{b})$ and $\mathrm{OD}(\mathrm{c})$ are the absorbances of the sample, blank and negative control, respectively.

Microscopy. At 24 and 48 h post-treatment, cells were visually monitored for morphological changes, such as lesions of the cell membrane and the compactness of cytoplasmic components under an inverted microscope (Bio Optical, Milan, Italy) at a magnification of $\mathrm{x} 200$.

Dose-dependent analysis of HBsAg expression in treated cells. HepG2.2.15 cells were seeded in 96-well plates $\left(0.5 \times 10^{5} /\right.$ well $)$ and incubated at $37^{\circ} \mathrm{C}$. The following day, the culture media was replaced with fresh media (150 $\mu 1$, each in triplicate) containing various doses $(0,6.25,12.5,25$ and $50 \mu \mathrm{g} / \mathrm{ml})$ of the test samples and controls, and incubated at $37^{\circ} \mathrm{C}$ for $48 \mathrm{~h}$. The culture supernatants of each sample were collected and stored at $-20^{\circ} \mathrm{C}$. The secreted HBsAg in the culture supernatants was analyzed using an ELISA kit (cat. no. 72348; Monolisa HBsAg ULTRA; Bio-Rad Laboratories Inc., Hercules, CA, USA) according to the manufacturer's instructions. OD was recorded using an ELx800 microplate reader and analyzed according to the manufacturer's instructions. Non-linear regression analysis was performed using Excel software to determine the half maximal (50\%) inhibitory concentration $\left(\mathrm{IC}_{50}\right)$ of $\mathrm{HBsAg}$ secretion. 
Table I. List of medicinal plants $(n=60)$ screened in the present study.

\begin{tabular}{|c|c|c|c|c|c|}
\hline No. & Plant name & Family & Part used & Voucher no. & Collection location \\
\hline 1 & Abutilon figarianum & Malvaceae & $\mathrm{L}$ & 16,082 & Riyadh, KSA \\
\hline 2 & Acacia hamulosa & Fabaceae & $\mathrm{L}+\mathrm{S}$ & 16,221 & South, KSA \\
\hline 3 & Acacia asak & Fabaceae & $\mathrm{L}$ & 16,387 & South, KSA \\
\hline 4 & Acacia ehrenbergiana & Fabaceae & $\mathrm{S}$ & 16,385 & South, KSA \\
\hline 5 & Acacia laeta & Fabaceae & $\mathrm{S}$ & 16,390 & South, KSA \\
\hline 6 & Acacia oerfota & Fabaceae & $\mathrm{S}$ & 16,389 & South, KSA \\
\hline 7 & Acacia salicina & Fabaceae & $\mathrm{L}$ & 15,007 & South, KSA \\
\hline 8 & Acacia tortilis & Fabaceae & $\mathrm{S}$ & 14,977 & South, KSA \\
\hline 9 & Achyranthes aspera & Amaranthaceae & Aerial parts $(\mathrm{S}, \mathrm{L}, \mathrm{Fr})$ & 16,011 & Gabeel, KSA \\
\hline 10 & Albizia procera & Fabaceae & $\mathrm{L}$ & 16,182 & Taif, KSA \\
\hline 11 & Alternanthera pungens & Amaranthaceae & Aerial parts $(\mathrm{S}, \mathrm{L}, \mathrm{Fr}, \mathrm{Fl})$ & 16,391 & South, KSA \\
\hline 12 & Amaranthus alba & Amaranthacea & Aerial parts $(\mathrm{S}, \mathrm{L}, \mathrm{Fr}, \mathrm{Fl})$ & 16,189 & Riyadh, KSA \\
\hline 13 & $\begin{array}{l}\text { Anagallis arvensis, } \\
\text { var. caerulea }\end{array}$ & Primulaceae & Aerial parts $(\mathrm{S}, \mathrm{L}, \mathrm{Fr}, \mathrm{Fl})$ & 16,296 & South, KSA \\
\hline 14 & Argemone ochroleuca & Papaveraceae & Aerial parts (S, L, Fr) & 16,185 & Taif, KSA \\
\hline 15 & Atriplex suberecta & Chenopodiaceae & Aerial parts $(\mathrm{S}, \mathrm{L}, \mathrm{Fr})$ & 16,195 & Taif, KSA \\
\hline 16 & Aerva Javanica & Amaranthaceae & Aerial parts $(\mathrm{S}, \mathrm{L}, \mathrm{Fl})$ & 16,196 & Taif, KSA \\
\hline 17 & Bacopa monieri & Scrophulariaceae & $\mathrm{L}+\mathrm{S}$ & 16,300 & South, KSA \\
\hline 18 & Balanites aegyptiaca & Zygophyllaceae & B & 560 & Khartoum, Sudan \\
\hline 19 & Boerhavia diffusa & Nyctaginaceae & $\mathrm{L}$ & 16,184 & Taif, KSA \\
\hline 20 & Bougainvillea spectabilis & Nyctaginaceae & $\mathrm{L}$ & 16,177 & Taif ,KSA \\
\hline 21 & Capparis decidua & Capparaceae & $\mathrm{S}$ & 15,841 & Tabouk, KSA \\
\hline 22 & Cassytha filiformis & Lauraceae & $\mathrm{S}$ & 15,716 & Taif, KSA \\
\hline 23 & Chenopodium ambrosioides & Chenopodiaceae & Aerial parts $(\mathrm{S}, \mathrm{L}, \mathrm{Fr})$ & 16,181 & Taif, KSA \\
\hline 24 & Chenopodiumg laucum & Chenopodiaceae & $\mathrm{L}+\mathrm{S}$ & 16,197 & Taif, KSA \\
\hline 25 & Citrus maxima & Rutaceae & $\mathrm{L}$ & 16,173 & Riyadh, KSA \\
\hline 26 & Cleome droserifolia & Crassulaceae & Aerial parts $(\mathrm{S}, \mathrm{L}, \mathrm{Fl})$ & 15,830 & Taif, KSA \\
\hline 27 & Clerodendrum inerme & Verbenaceae & $\mathrm{L}+\mathrm{S}$ & 12,788 & Riyadh, KSA \\
\hline 28 & Coccinia grandis & Cucurbitaceae & $\mathrm{L}+\mathrm{S}$ & 16,275 & South, KSA \\
\hline 29 & Combretum molle & Combretaceae & $\mathrm{B}$ & 15,496 & South, KSA \\
\hline 30 & Corallocarpus epigeus & Cucurbitaceae & $\mathrm{L}$ & 16,393 & South, KSA \\
\hline 31 & Daturai noxia & Solanaceae & $\mathrm{L}$ & 15,604 & Riyadh, KSA \\
\hline 32 & Delonix elata & Fabaceae & $\mathrm{L}$ & 16,035 & South, KSA \\
\hline 33 & Delonix regia & Fabaceae & $\mathrm{L}$ & 16,183 & Taif, KSA \\
\hline 34 & Dodonea angustifolia & Sapindaceae & $\mathrm{L}$ & 15,787 & South, KSA \\
\hline 35 & Eruca sativa & Brassicaceae & $\mathrm{L}+\mathrm{S}$ & 16,318 & South, KSA \\
\hline 36 & Euphorbia tirucalli & Euphorbiaceae & $\mathrm{S}$ & 16,172 & Riyadh, KSA \\
\hline 37 & Euphorbia hirta & Euphorbiaceae & Aerial parts $(\mathrm{S}, \mathrm{L}, \mathrm{Fr})$ & 16,084 & Omdurman, Sudan \\
\hline 38 & Ficus benghalensis & Moraceae & $L+B$ & 16,080 & Riyadh, KSA \\
\hline 39 & Ficus palmata & Moraceae & $\mathrm{L}$ & 15,448 & Tanoma, KSA \\
\hline 40 & Flaveria trineriva & Asreraceae & Arial parts $(\mathrm{S}, \mathrm{L}, \mathrm{Fr})$ & 16,198 & Taif, KSA \\
\hline 41 & Fumaria parviflora & Fumariaceae & $\mathrm{L}+\mathrm{S}$ & 16,301 & South, KSA \\
\hline 42 & Guiera senegalensis & Combretaceae & $\mathrm{L}$ & 798 & Kordofan, Sudan \\
\hline 43 & Haplophyllum tuberculum & Rutaceae & Aerial parts $(\mathrm{S}, \mathrm{L}, \mathrm{Fl})$ & 16,324 & South, KSA \\
\hline 44 & Indigofera caerulea & Fabaceae & Aerial parts $(\mathrm{S}, \mathrm{L}, \mathrm{Fl})$ & 16,392 & South, KSA \\
\hline 45 & Ipomoea cairica (L.) sweet & Convolvulaceae & Aerial parts $(\mathrm{S}, \mathrm{L}, \mathrm{Fr})$ & 16,075 & Riyadh, KSA \\
\hline 46 & Indigofera tinctoria & Fabaceae & Aerial parts $(\mathrm{S}, \mathrm{L}, \mathrm{Fr})$ & 16,390 & South, KSA \\
\hline 47 & Jatropha curcas & Euphorbiaceae & Seeds & 15,189 & Riyadh, KSA \\
\hline 48 & Juniperus phonicea & Cupressaceae & $\mathrm{S}+\mathrm{L}$ & 16,179 & Taif, KSA, \\
\hline 49 & Juniperus procera & Cupressaceae & $\mathrm{S}+\mathrm{L}$ & 16,194 & Taif KSA, \\
\hline 50 & Marrubium vulgare & Labiatae & Aerial parts $(\mathrm{S}, \mathrm{L}, \mathrm{Fr})$ & 16,043 & Hadah, KSA \\
\hline 51 & Momordica balsamina & Cucurbitaceae & $\mathrm{L}$ & 16,395 & South, KSA \\
\hline
\end{tabular}


Table I. Continued.

\begin{tabular}{|c|c|c|c|c|c|}
\hline No. & Plant name & Family & Part used & Voucher no. & Collection location \\
\hline 52 & Pergularia tomentosa & Asclepiadaceae & Aerial parts $(\mathrm{S}, \mathrm{L}, \mathrm{Fr})$ & 16,075 & Riyadh, KSA \\
\hline 53 & Psidiumg uajava & Myrtaceae & $\mathrm{L}$ & 16,085 & South, KSA \\
\hline 54 & Pulicaria crispa & Asteraceae & Aerial parts $(\mathrm{S}, \mathrm{L}, \mathrm{Fr})$ & 16,083 & Riyadh, KSA \\
\hline 55 & Ricinus communis & Euphorbiaceae & $\mathrm{L}$ & 14,005 & Riyadh, KSA \\
\hline 56 & Rumex dentatus & Polygonaceae & Aerial parts $(\mathrm{S}, \mathrm{L}, \mathrm{Fr})$ & 16,186 & Taif, KSA \\
\hline 57 & Senna obtusifolia & Fabaceae & $\mathrm{Fr}$ & 160,322 & South, KSA \\
\hline 58 & Senna occidentalis & Fabaceae & $\mathrm{Fr}$ & 155,009 & South, KSA \\
\hline 59 & Senna alexandrina & Fabaceae & $\mathrm{L}$ & 16,245 & South, KSA \\
\hline 60 & Solanum surrattense & Solanaceae & $\mathrm{L}$ & 16,386 & South, KSA \\
\hline
\end{tabular}

L, leaves; S, stems; B, bark; Fr, fruits; KSA, Kingdom of Saudi Arabia.

Time-course analysis of HBsAg inhibition. Based on the dose-dependent inhibition results, time-course (day 1,3 and 5) analyses were performed to further investigate the antiviral potential of the most active extracts. The HBsAg expression study was performed by treating cells with the safest single-dose $(50 \mu \mathrm{g} / \mathrm{ml})$, as determined by the $\mathrm{IC}_{50}$ values.

Time-course analysis of HBeAg inhibition. Extracts exhibiting the most promising inhibitory effects on HBsAg secretions were further subjected to time-course (day 1, 3 and 5) analyses of $\mathrm{HBeAg}$ expression at a dose of $50 \mu \mathrm{g} / \mathrm{ml}$. ELISA was performed on the culture media using an $\mathrm{HBeAg} / \mathrm{anti}-\mathrm{HBe}$ ELISA kit (cat. no. KAPG4BNE3; DIAsource ImmunoAssays; SA, Louvain-la-Neuve, Belgium) according to the manufacturer's instructions. OD was recorded using an ELx800 microplate reader, and analyzed following the DIASource manual.

Phytochemical constituent screening. Plants exhibiting the most promising anti-HBV activities were subjected to qualitative phytochemical screening for major secondary metabolites, including alkaloids, flavonoids, anthraquinones, tannins and saponins, following standard procedures (15-17) with minor modifications. Briefly, for alkaloids, the Mayer's test was performed. A total of $0.5 \mathrm{gm}$ of the extract was dissolved in $2 \%$ hydrochloric acid (Sigma-Aldrich; Merck KGaA), and filtered. Mayer's reagent was freshly prepared by dissolving $0.68 \mathrm{~g}$ of mercuric chloride (Sigma-Aldrich; Merck KGaA) and $2.5 \mathrm{~g}$ of potassium iodide (Sigma-Aldrich; Merck KGaA) and made to $50 \mathrm{ml}$ with distilled water. A few drops of the reagent were added to the $3 \mathrm{ml}$ of extract solution in a test tube where formation of a yellowish precipitate indicated the presence of alkaloids. For flavonoids, the sodium hydroxide test was performed. A total of $5 \mathrm{ml}$ of the extract solution was treated with few drops of $20 \%$ sodium hydroxide (Sigma-Aldrich; Merck KGaA) in a test tube. Formation of an intense yellow color, which becomes colorless on addition of diluted hydrochloric acid, indicated the presence of flavonoids. For tannins, the ferric chloride test was performed. A total of $0.25 \mathrm{gm}$ of the extract was dissolved in $10 \mathrm{ml}$ of distilled water in a test tube and few drops of 5\% ferric chloride (Sigma-Aldrich;
Merck KGaA) was added. Appearance of brownish green or blueish-black color indicated the presence of tannins. For saponins, the frothing test was performed. A total of $0.5 \mathrm{gm}$ of the extract was dissolved in $10 \mathrm{ml}$ of distilled water in a test tube and shaken vigorously. Formation of a thick persistent froth that persisted for at least 15 min indicated the presence of saponins. And lastly, for anthraquinone the Borntrager's test was performed. A total $0.5 \mathrm{gm}$ of the extract residue was boiled with $5 \mathrm{ml}$ of dilute hydrochloric acid and filtered while hot. The filtrate was combined with $5 \mathrm{ml}$ of chloroform and shaken. The chloroform layer was transferred into a test tube and $2 \mathrm{ml}$ of dilute ammonia solution (Sigma-Aldrich; Merck $\mathrm{KGaA}$ ) was added. The appearance of a rose-pink to cherry-red color indicated the presence of anthraquinones.

\section{Results}

Effects of plants extracts on cell viability. $\mathrm{CC}_{50}$ values of different plants extracts were calculated (Table II), which allowed for the determination of the single optimal dose $(50 \mu \mathrm{g} / \mathrm{ml})$ with no sign of cytotoxicity. This observation was confirmed by microscopic observation of the cell morphology/growth at 24 and $48 \mathrm{~h}$ post-treatment with different concentrations of each extract (data not shown). Therefore, extracts at $50 \mu \mathrm{g} / \mathrm{ml}$ were used in the subsequent antiviral assays.

Dose-dependent inhibition of HBsAg by different extracts. Firstly, the total ethanolic-extracts of 60 medicinal plants were screened for anti-HBV activity by measuring the expression levels of viral HBsAg at $48 \mathrm{~h}$. Of these, 9 plants showed inhibition of HBsAg production in a dose-dependent manner. These were Abutilon figarianum, Acacia oerfota, Capparis decidua, Coccinea grandis, Corallocarpus epigeus, Fumaria parviflora, Guiera senegalensis, Indigofera caerulea and Pulicaria crispa with $\mathrm{IC}_{50}$ values of 106.46, 101.46, 76.85, 31.57, 71.90, $79.84,73.21,84.62$ and $23.10 \mu \mathrm{g} / \mathrm{ml}$, respectively (Table II). Based on these results, 5 sequential extracts (hexane, dichloromethane, ethyl acetate, methanol and aqueous) of each of the 9 selected plants were prepared, and tested for cytotoxicity. The sequential extracts were further evaluated for dose-dependent 
Table II. Determination of cytotoxicity concentration $\left(\mathrm{CC}_{50}\right)$ and anti-hepatitis B virus activity (inhibition of HBsAg secretion via $\mathrm{IC}_{50}$ ) and the corresponding TI of the plant extracts.

\begin{tabular}{|c|c|c|c|c|c|}
\hline Plant name & Extraction solvent & Yield (\%) & $\mathrm{CC}_{50}(\mu \mathrm{g} / \mathrm{ml})$ & $\mathrm{IC}_{50}(\mu \mathrm{g} / \mathrm{ml})$ & $\mathrm{TI}$ \\
\hline \multirow[t]{6}{*}{ Abutilon figarianum } & $\mathrm{EtOH}$ & 8.71 & 366.67 & 106.46 & 3.44 \\
\hline & Hex & 1.10 & 1700.00 & NA & ND \\
\hline & DCM & 0.64 & 1375.02 & 99.76 & 13.78 \\
\hline & EtAc & 0.48 & 332.30 & NA & ND \\
\hline & $\mathrm{MeOH}$ & 8.02 & 284.70 & NA & ND \\
\hline & Aqua & 2.21 & $\mathrm{NC}$ & NA & ND \\
\hline \multirow[t]{6}{*}{ Acacia oerfota } & $\mathrm{EtOH}$ & 11.13 & 1375.00 & 101.46 & 13.55 \\
\hline & Hex & 2.64 & 1150.10 & NA & ND \\
\hline & DCM & 0.19 & 960.00 & NA & ND \\
\hline & EtAc & 0.79 & $\mathrm{NC}$ & NA & ND \\
\hline & $\mathrm{MeOH}$ & 5.54 & 383.30 & 118.90 & 3.22 \\
\hline & Aqua & 9.60 & 422.20 & 106.84 & 3.95 \\
\hline \multirow[t]{6}{*}{ Capparis decidua } & $\mathrm{EtOH}$ & 10.31 & 366.67 & 76.85 & 4.77 \\
\hline & Hex & 0.27 & 383.30 & NA & ND \\
\hline & DCM & 0.51 & 400.00 & NA & ND \\
\hline & EtAc & 0.18 & 833.10 & NA & ND \\
\hline & $\mathrm{MeOH}$ & 3.86 & 1667.67 & NA & ND \\
\hline & Aqua & 6.86 & 520.00 & 66.82 & 7.78 \\
\hline \multirow[t]{6}{*}{ Coccinia grandis } & $\mathrm{EtOH}$ & 8.71 & 219.44 & 31.57 & 6.95 \\
\hline & Hex & 1.33 & 800.00 & NA & ND \\
\hline & DCM & 0.86 & 480.01 & 57.14 & 8.40 \\
\hline & EtAc & 0.32 & $\mathrm{NC}$ & NA & ND \\
\hline & $\mathrm{MeOH}$ & 7.28 & 800 & NA & ND \\
\hline & Aqua & 5.61 & 557.14 & 87.21 & 6.38 \\
\hline \multirow[t]{6}{*}{ Corallocarpus epigeus } & $\mathrm{EtOH}$ & 7.35 & 1275.00 & 71.90 & 17.73 \\
\hline & Hex & 0.86 & 150.00 & NA & ND \\
\hline & $\mathrm{DCM}$ & 0.50 & 112.90 & NA & ND \\
\hline & EtAc & 0.31 & 153.37 & NA & ND \\
\hline & $\mathrm{MeOH}$ & 0.72 & 2500.00 & NA & ND \\
\hline & Aqua & 2.61 & 1094.00 & 70.91 & 15.42 \\
\hline \multirow[t]{6}{*}{ Fumaria parviflora } & $\mathrm{EtOH}$ & 9.35 & $\mathrm{NC}$ & 79.84 & ND \\
\hline & Hex & 0.88 & 425.00 & 35.44 & 11.99 \\
\hline & DCM & 0.62 & 188.10 & NA & ND \\
\hline & EtAc & 1.02 & 221.98 & NA & ND \\
\hline & $\mathrm{MeOH}$ & 8.10 & 1950.00 & 79.19 & 24.62 \\
\hline & Aqua & 2.61 & 766.67 & $\mathrm{NA}$ & ND \\
\hline \multirow[t]{6}{*}{ Guiera senegalensis } & $\mathrm{EtOH}$ & 9.76 & 1566.00 & 73.21 & 21.39 \\
\hline & Hex & 0.52 & 3330.00 & NA & ND \\
\hline & DCM & 0.74 & 200.00 & 10.65 & 18.77 \\
\hline & EtAc & 0.62 & 450.10 & NA & ND \\
\hline & $\mathrm{MeOH}$ & 7.32 & 1000.06 & NA & ND \\
\hline & Aqua & 2.08 & 370.69 & 76.67 & 4.83 \\
\hline \multirow[t]{6}{*}{ Indigofera caerulea } & $\mathrm{EtOH}$ & 8.61 & 1455.00 & 84.62 & 17.19 \\
\hline & Hex & 0.62 & 230.43 & NA & ND \\
\hline & DCM & 0.66 & 208.00 & NA & ND \\
\hline & EtAc & 0.42 & 642.80 & NA & ND \\
\hline & $\mathrm{MeOH}$ & 2.47 & 1566 & 73.21 & 21.39 \\
\hline & Aqua & 4.06 & 1250.00 & 125.10 & 9.99 \\
\hline \multirow[t]{2}{*}{ Pulicaria crispa } & $\mathrm{EtOH}$ & 12.17 & 686.71 & 23.10 & 29.72 \\
\hline & Hex & 1.24 & 160.10 & NA & ND \\
\hline
\end{tabular}


Table II. Continued.

\begin{tabular}{|c|c|c|c|c|c|}
\hline Plant name & Extraction solvent & Yield $(\%)$ & $\mathrm{CC}_{50}(\mu \mathrm{g} / \mathrm{ml})$ & $\mathrm{IC}_{50}(\mu \mathrm{g} / \mathrm{ml})$ & $\mathrm{TI}$ \\
\hline \multirow[t]{4}{*}{ Pulicaria crispa } & DCM & 1.64 & 79.31 & NA & ND \\
\hline & EtAc & 0.48 & 603.00 & 14.45 & 41.04 \\
\hline & $\mathrm{MeOH}$ & 7.80 & 258.33 & 141.72 & 1.82 \\
\hline & Aqua & 4.41 & 733.30 & NA & ND \\
\hline
\end{tabular}

$\mathrm{CC}_{50}, 50 \%$ cytotoxicity concentration; $\mathrm{IC}_{50}$, half maximal inhibitory concentration; Hex, hexane; DCM, dichloromethane; EtOAc, ethyl acetate; $\mathrm{MeOH}$, methanol; Aqua, water; NC, non-cytotoxic; NA, not active; TI, therapeutic index; ND, not determined.

A

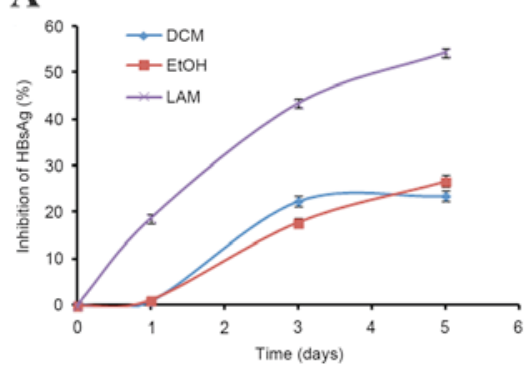

D

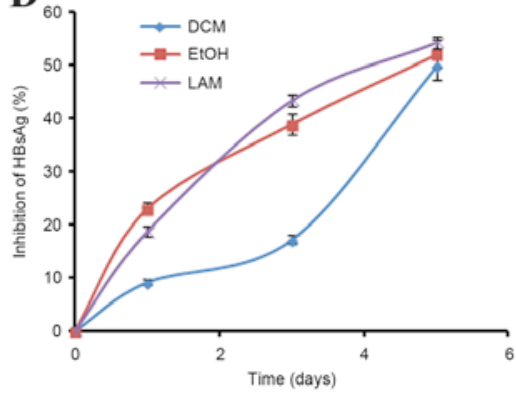

G

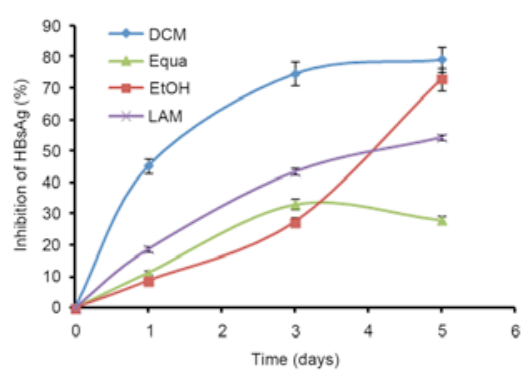

B

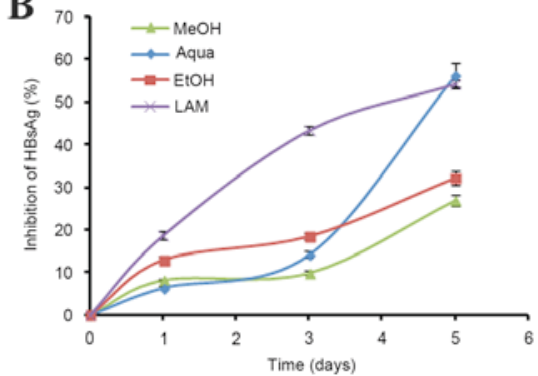

$\mathbf{E}$

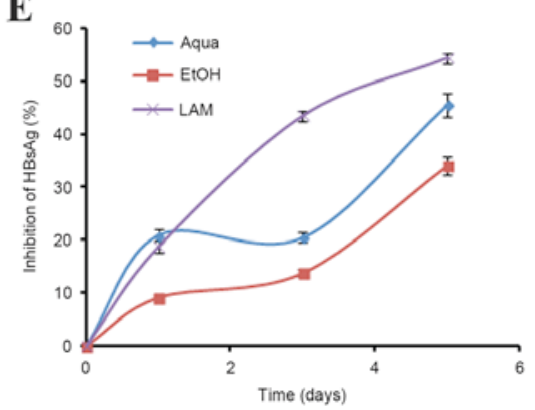

H

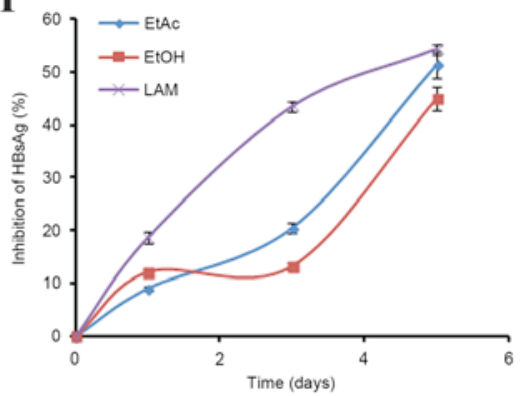

C

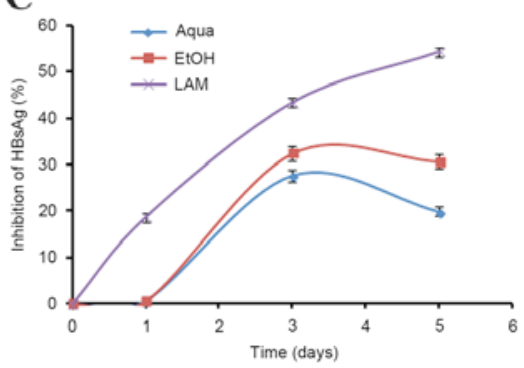

$\mathbf{F}$

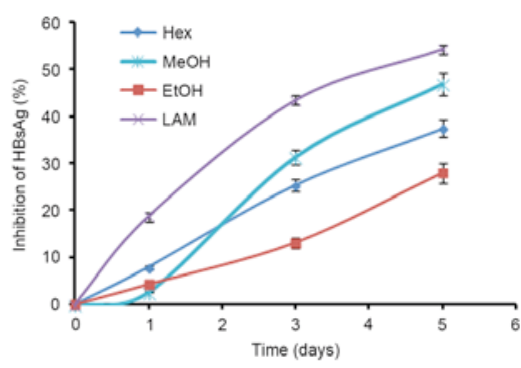

I

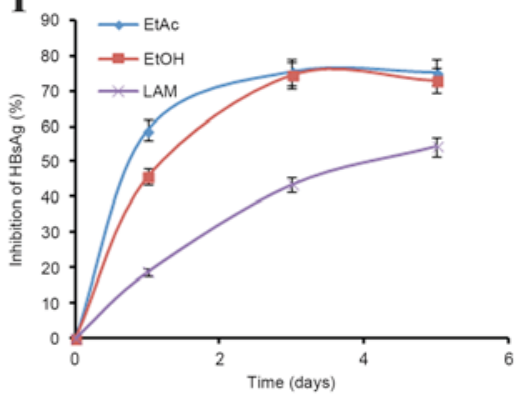

Figure 1. Time-course anti-HBV activity of selected plant extracts (50 $\mu \mathrm{g} / \mathrm{ml}$ each). (A) Abutilon figarianum; (B) Acacia oerfota; (C) Capparis decidua; (D) Co ccinia grandis; (E) Corallocarpus epigeus; (F) Fumaria parviflora; (G) Guiera senegalensis; (H) Indigofera caerulea; (I) Pulicaria crispa. ELISA showing inhibitions of HBsAg expression in HepG2.2.15 culture supernatants at days 1, 3 and 5 post-treatment. Lamivudine $(2.0 \mu \mathrm{M})$ was used as a reference anti-HBV drug. Values (y-axis): means of three determinations. HBV, hepatitis B virus; Aqua, aqueous; DCM, dichloromethane; EtAc, ethyl acetate; EtOH, ethanol; Hex, hexane; $\mathrm{MeOH}$, methanol; LAM, lamivudine.

HBsAg inhibition. The extraction yield percentage, $\mathrm{IC}_{50}, \mathrm{CC}_{50}$ and their corresponding TI values were calculated (Table II). Of these, 24 different extracts (from the 9 selected plants) that showed marked HBsAg inhibition were evaluated in a time-course study.

Time-course inhibition of HBsAg by selected extracts. The selected extracts (of 9 plants) that showed marked HBsAg inhibition were evaluated in a time-course study, using $50 \mu \mathrm{g} / \mathrm{ml}$ doses for 5 days (Fig. 1). While prolonged treatment beyond day 5 did not show any notable inhibitory effect, further continuation of the culture resulted in cell overgrowth and death (data not shown). The optimal antiviral activities, in order, on day 5 post-treatment were: $G$. senegalensis (dichloromethane extract; $\mathrm{IC}_{50}=10.65$ ), $P$. crispa (ethyl acetate extract; $\mathrm{IC}_{50}=14.45$ ), $C$. gardis (total ethanol extract; $\left.\mathrm{IC}_{50}=31.57\right)$, F. parviflora (hexane extract; $\left.\mathrm{IC}_{50}=35.44\right), C$. decidua (aqueous extract; $\mathrm{IC}_{50}=66.82$ ), C. epigeus (total ethanol extract; $\mathrm{IC}_{50}=71.9$ ), $I$. caerulea (methanol extract; $\mathrm{IC}_{50}=73.21$ ), 
Table III. Qualitative phytochemical screening of plants with anti-hepatitis B virus activities.

Phytochemicals

\begin{tabular}{|c|c|c|c|c|c|}
\hline \multirow[b]{2}{*}{ Active plant extracts } & & & & & \\
\hline & Alkaloids & Flavonoids & Tannins & Saponins & Anthraquinones \\
\hline
\end{tabular}

\begin{tabular}{|c|c|c|c|c|c|}
\hline Abutilon figarianum & + & - & - & - & - \\
\hline Acacia oerfota & + & + & + & - & - \\
\hline Capparis deciduas & + & + & + & + & - \\
\hline Coccinea grandis & + & + & + & + & - \\
\hline Corallocarpus epigeus & + & + & + & - & - \\
\hline Fumaria parviflora & + & + & + & + & - \\
\hline Guiera senegalensis & + & + & + & + & - \\
\hline Indigofera caerulea & + & + & + & + & - \\
\hline Pulicaria crispa & + & + & + & - & - \\
\hline
\end{tabular}

+ , detected; -, not detected.

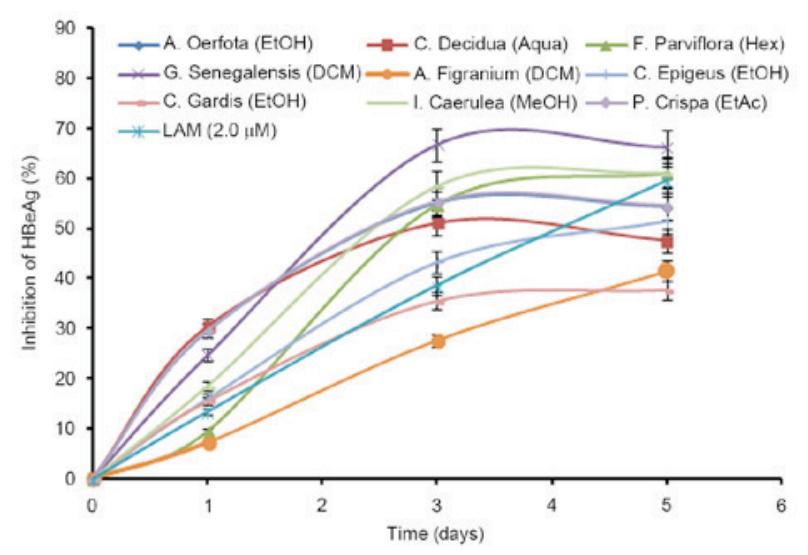

Figure 2. Time-course anti-HBV activity of the plants extracts with anti-HBV potential (50 $\mu \mathrm{g} / \mathrm{ml}$ each). ELISA showing inhibitions of HBeAg expression in HepG2.2.15 culture supernatants at days 1, 3 and 5 post-treatment. Lamivudine $(2.0 \mu \mathrm{M})$ was used as a reference anti-HBV drug. Values (y-axis): means of three determinations. HBV, hepatitis B virus; Aqua, aqueous; DCM, dichloromethane; EtAc, ethyl acetate; EtOH, ethanol; Hex, hexane; $\mathrm{MeOH}$, methanol; LAM, lamivudine.

A. figarianum (dichloromethane extract; $\mathrm{IC}_{50}=99.76$ ) and $A$. oerfota (total ethanol extract; $\mathrm{IC}_{50}=101.46$ ) (Table II).

Time-course downregulation of $H B V$ replication by the active extracts. The HBeAg is a processed product of the 'pre-Core' gene that is co-translated with the 'Core' gene by bicistronic subgenomic-RNA. Therefore, in a natural infection, seropositivity of $\mathrm{HBeAg}$ is a hallmark of active viral DNA replication. Notably, this is analogous to the HIV 'p24' antigen where ELISA is a valid tool to monitor retroviral RNA replication. Therefore, the most promising active extracts that greatly suppressed HBsAg synthesis (Fig. 1), were analyzed for time-course effect on $\mathrm{HBeAg}$ production in the culture supernatants. While $\mathrm{HBeAg}$ secretion was inhibited maximally at day 3, there were no further improvements in antiviral activities at day 5 (Fig. 2). Limited by cell overgrowth and death, and unaffected virus replication (inhibition of $\mathrm{HBeAg}$ ), the study was terminated at day 5. Furthermore, the antiviral activities on downregulating virus replication, as measured by $\mathrm{HBeAg}$ production, were: $G$. senegalensis (dichloromethane extract; 66\%); I. caerulea (methanol extract; $58.60 \%)$; P. crispa (ethyl acetate extract; $55.30 \%$ ); F. parviflora (hexane-extract; $54.68 \%$ ); C. decidua (aqueous extract; 51.52\%); C. epigeus (total ethanol extract; 43.31\%); $C$. grandis (total ethanol extract; 35.51\%); A. oerfota (total ethanol extract; 35.28\%); and A. figarianum (dichloro methane extract; $27.56 \%$ ) compared to the untreated control (Fig. 2). It is noteworthy that, with the exception of $C$.grandis and A.figarianum, all plant extracts $(50 \mu \mathrm{g} / \mathrm{ml})$ showed greater antiviral activity than lamivudine $(2 \mu \mathrm{M})$.

Phytochemical screening. Plants that exhibited anti-HBV activity showed the presence of alkaloids, flavonoids, tannins, saponins and anthraquinones (Table III), which have been previously reported for their antiviral activities $(7,8)$.

\section{Discussion}

Development of anti-HBV therapies has been impeded until recently by the lack of suitable in vitro and in vivo experimental models that were able mimic natural chronic hepatitis B (18-21). Several lines of hepatoma cells stably transfected with HBV genome have been developed as an in vitro model to screen and identify potential antiviral therapeutic agents. Of these, in the present study, the widely used Hep G2.2.15 cells were used to evaluate the anti-HBV potential of candidate plants by measuring the expression of HBsAg (serological marker of viral infection) and HBeAg (serological marker of active DNA replication), respectively $(22,23)$. A total of 60 medicinal plants were investigated for the first time, based on information on either their use in traditional medicine for curing liver diseases or experimental evidence of hepatoprotective or anti-retroviral potentials $(12,13,24-37)$. The preliminary cell viability assay of the plants' total ethanolic extracts in the present study showed no cytotoxicity at concentrations up to $50 \mu \mathrm{g} / \mathrm{ml}$. Further initial screening (dose-dependent HBsAg inhibition) identified 9 plants with notable anti-HBV activity that were therefore selected for sequential extractions (organic and aqueous phase) and subsequent screening. 
The highest level of anti-HBV activity was observed in the dichloromethane extract of $G$. senegalensis, known as the 'cure all' medicine in African traditional medicine due to its wide applications (24). It is used to treat venereal, stomach, respiratory, dermatological and microbial diseases (25), including malaria (26). In agreement with its reported anti-HSV potential (27), G. senegalensis is likely to have exhibited anti-HBV activity because HSV and HBV are biologically and genetically similar. In addition to this, it has previously been reported that administration of $G$. senegalensis extract to Wistar rats for 6 months did not cause any significant hematological, biochemical or histological toxicity (28), confirming its in vivo safety. Assuming there was no synergy among the phytochemical constituents present in the dichloromethane-extract, it can be implied that the active compound(s) could be more potent than the lamivudine used as standard reference drugs.

$F$. parviflora has traditionally been used in Saudi folk medicine for the treatment of jaundice and hepatobiliary disorders (29). At day 5 post-treatment, hexane and methanol extracts of $F$. parviflora showed the best anti-HBV activities by $\sim 37.45$ and $46.86 \%$, respectively. Besides its use in Sudanese traditional medicine to treat fever and jaundice, an aqueous-extract of $C$. decidua has been demonstrated to show hepatoprotective activity in rats (30). Notably, in line with its reported antiviral activity against HIV reverse-transcriptase (31), the aqueous-extract of $C$. decidua exhibited anti-HBV potential in the present study.

Acacia spp. constitute a large variety of medicinal plants worldwide, and of these, A. catechu has been previously reported for its anti-HIV activity (23). The present authors have previously demonstrated that A. mellifera ethyl acetate, n-butanol and aqueous extracts also have hepatoprotective and anti-HBV effects (32). In the present study, anti-HBV evaluation of $A$. oerfota extracts at a non-cytotoxic dose showed its association with the methanolic and aqueous extracts. Furthermore, the highest anti-HBV activity of $C$. grandis and $C$. epigeus was associated with the crude ethanolic-extract, indicating the possibility of synergy among the antiviral phytochemical constituents of the extract. Synergistic activity of antiviral components of plant extracts that act by different mechanisms has been reported previously (33). Traditionally, crude extracts of $C$. grandis are used to treat coughing, bronchitis, skin diseases, tongue sores and liver disorders (34). In previous studies, in vivo antioxidant and hepatoprotective efficacies of C. grandis (crude ethanolic-extract) and C. epigeus (ethanolic and aqueous extracts) have been demonstrated (35-37).

A variety of active phytochemicals (alkaloids, flavonoids, lignans, tannins, terpenoids, saponins and anthraquinones) of diverse geographic origins have already been reported to be effective against $\mathrm{HBV}$ in vitro and/or in vivo $(7,8,38,39)$. Of these, promising anti-HBV phytoproducts such as picroliv (Picrorhiza kurroa), andrographolide (Andrographis paniculata), artemisinin (Artemisia annua) and silymarin have been reported for a long time (40). Notably, the most potent anti-HBV phytochemicals include isolated niranthin and hinokinin (lignans). From Phyllanthus spp. (41-43), helioxanthin from the Chinese Taiwania cryptomerioides (44), wogonin, another flavonoid from Scutellaria radix (45), the polyphenolic extract from Geranium carolinianum L. (46), protostane triterpenes from Alisma orientalis (47), dihydrochelerythrine alkaloids from Corydalis saxicola (48), Saikosaponin C from Bupleurum species (49), extracts from Rheum palmatum L. (50), and LPRP from Liriope platyphylla (51). Furthermore, the qualitative phytochemical analyses of the selected plant extracts in the present study that showed promising anti-HBV potential also revealed the presence of alkaloids, flavonoids, triterpenoids and tannin, which may have contributed to the antiviral activities observed. However, a detailed phytochemical investigation of these extracts is essential to elucidate the active principle(s) responsible for the anti-HBV potential.

In conclusion, antiviral screening in the present study discovered that extracts of G. senegalensis, F. parviflora and $P$. crispa had the most promising anti-HBV potential, followed by those of A. figarianum, A. oerfota, C. decidua, C. grandis, C. epigeus and I. caerulea with notable activities. From the results of the present study, it is possible to demonstrate the importance of the application of ethnobotanical information in the search and selection of traditionally used plants, which may provide new opportunities for the treatment of chronic hepatitis B. However, a detailed phytochemical study of these extracts is required in order to elucidate the active principle(s) responsible for their novel anti-HBV potential.

\section{Acknowledgements}

The project was supported by the National Plan for Science, Technology and Innovation (MARIFAAH) funded by King Abdulaziz City for Science and Technology, Kingdom of Saudi Arabia (grant no. MED11-1585-02).

\section{References}

1. Teo CG and Locarnini SA: Potential threat of drug-resistant and vaccine-escape HBV mutants to public health. Antivir Ther 15: 445-449, 2010.

2. Torresi J: Hepatitis B antiviral resistance and vaccine escape: Two sides of the same coin. Antivir Ther 13: 337-340, 2008.

3. Lok AS, Zoulim F, Locarnini S, Bartholomeusz A, Ghany MG Pawlotsky JM, Liaw YF, Mizokami M and Kuiken C; Hepatitis B Virus Drug Resistance Working Group: Antiviral drug-resistant HBV: Standardization of nomenclature and assays and recommendations for management. Hepatology 46: 254-265, 2007.

4. Locarnini S: Primary resistance, multidrug resistance, and cross-resistance pathways in HBV as a consequence of treatment failure. Hepatol Int 2: 147-151, 2008

5. Chen Y and Zhu J: Anti-HBV effect of individual traditional chinese herbal medicine in vitro and in vivo: An analytic review. J Viral Hep 20: 445-452, 2013.

6. Qiu LP and Chen KP: Anti-HBV agents derived from botanical origin. Fitoterapia 84: 140-157, 2013.

7. Parvez MK, Arbab AH, Al-Dosari MS and Al-Rehaily AJ: Antiviral natural products against chronic hepatitis B: Recent developments. Curr Pharm Des 22: 286-293, 2016.

8. Wu YH: Naturally derived anti-hepatitis B virus agents and their mechanism of action. World J Gastroenterol 22: 188-204, 2016.

9. Chen HC, Chou CK, Lee SD, Wang JC and Yeh SF: Active compounds from Saussurea lappa Clarks that suppress hepatitis $B$ virus surface antigen gene expression in human hepatoma cells. Antiviral Res 27: 99-109, 1995.

10. Chou SC, Huang TJ, Lin EH, Huang $\mathrm{CH}$ and Chou $\mathrm{CH}$ : Anti-hepatitis B virus constituents from Solanum erianthum. Nat Prod Commun 78: 153-156, 2012.

11. Li J, Huang H, Zhou W, Feng M and Zhou P: Anti-hepatitis B virus activities of Geranium carolinianum L. extracts and identification of the active components. Biol Pharma Bull 31: 743-747, 2008. 
12. Rahman MA, Mossa JS, Al-Said MS and Al-Yahya MA: Medicinal plant diversity in the flora of Saudi Arabia 1: A report on seven plant families. Fitoterapia 75: 149-161, 2004.

13. Al-Asmari AK, Al-Elaiwi AM, Athar MT, Tariq M, Al Eid A and Al-Asmary SM: A review of hepatoprotective plants used in saudi traditional medicine. Evid Based Complement Alternat Med. 2014: 890842, 2014

14. van Meerloo J, Kaspers GJ and Cloos J: Cell sensitivity assays: The MTT assay. Methods Mol Biol 731: 237-245, 2011.

15. Satyajit D, Sarkar ZL and Gray AI: Natural Products Isolation. Humana Press, Totowa, NJ, USA, 2nd edition, 2006.

16. Kar A: Pharmacognosy and Pharmacobiotechnology. New Age International, New Delhi, India, 2nd edition, 2007.

17. Tiwari P, Mandeep BK, Kaur KG and Kaur H: Phytochemical screening and extraction: A review. Interl Pharma Sci 1: 98-1062, 2011.

18. Hantz O and Zoulim F: Duck hepatitis B virus primary hepatocyte culture model. Methods Mol Med 96: 189-197, 2004

19. Engler OB, Dai WJ, Sette A, Hunziker IP, Reichen J, Pichler WJ and Cerny A: Peptide vaccines against hepatitis b virus: From animal model to human studies. Mol Immunol 38: 457-465, 2001.

20. Thung SN, Gerber MA, Purcell RH, London WT, Michalik KB and Popper H: Animal model of human disease: Chimpanzee carriers of hepatitis B virus. Chimpanzee hepatitis B carriers Am J Pathol 105: 328-332, 1981.

21. Aljofan M, Netter HJ, Aljarbou AN, Hadda TB, Orhan IE, Sener B and Mungall BA: Anti-hepatitis B activity of isoquinoline alkaloids of plant origin. Arch Virol 159: 1119-1128, 2014.

22. Bonino F, Hoyer B, Nelson J, Engle R, Verme G and Gerin J: Hepatitis B virus DNA in the sera of HBsAg carriers: A marker of active hepatitis B virus replication in the liver. Hepatology 1 : 386-391, 1981 .

23. Arbab AH, Parvez MK, Al-Dosari MS, Al-Rehaily AJ, Al-Sohaibani M, Zaroug EE, AlSaid MS and Rafatullah S: Hepatoprotective and antiviral efficacy of Acacia mellifera leaves extracts against hepatitis B virus. Biomed Res Int 2015: 929131, 2015

24. Akuodor GC, Essien AD, David-Oku E, Chilaka KC, Akpan JL, Ezeokpo B and Ezeonwumelu JO: Gastroprotective effect of the aqueous leaf extract of Guiera senegalensis in albino rats. Asian Pacific J Trop Med 6: 771-775, 2013.

25. Silva O, Serrano R and Gomes ET: Botanical characterization of Guiera senegalensis leaves. Microsc Microanal 14: 398-404, 2008 .

26. Abubakar MS, Sule MI, Pateh UU, Abdurahman EM, Haruna AK and Jahun BM: In vitro snake venom detoxifying action of the leaf extract of Guiera senegalensis. J Ethnopharmacol 69 : 253-257, 2000

27. Silva O, Barbosa S, Diniz A, Valdeira ML and Gomes E: Plant extracts antiviral activity against herpes simplex virus type 1 and African swine fever virus. Interl J Pharmacog 35: 12-16, 1997.

28. Diouf A, Cisse A, Gueye SS, Mendes V, Siby T, Diouf Diop RM and Bassene E: Toxocological study of Guiera senegalensis Lam (Combretaceae). Dakar Med 45: 89-94, 2000 (In French).

29. Najeeb-ur-Rehman, Bashir S, Al-Rehaily AJ and Gilani AH: Mechanisms underlying the antidiarrheal, antispasmodic and bronchodilator activities of Fumaria parviflora and involvement of tissue and species specificity. J Ethnopharmacol 144: 128-137, 2012.

30. Ali H, König GM, Khalid SA, Wright AD and Kaminsky R: Evaluation of selected Sudanese medicinal plants for their in vitro activity against hemoflagellates, selected bacteria, HIV-RT and tyrosine kinase inhibitory, and for cytotoxicity. J Ethnopharmacol 83: 219-228, 2002.

31. Ali SA, Gameel AA, Mohamed AH and Hassan $\mathrm{T}$ : Hepatoprotective activity of Capparis decidua aqueous and methanolic stems extracts against carbon tetrachloride induced liver histological damage in rats. J Pharmacol Toxicol 6: 62-68, 2011.

32. Nutan M, Modi C, Dezzutti CS, Kulshreshtha S, Rawat AK, Srivastava SK, Malhotra S, Verma A, Ranga U and Gupta SK: Extracts from Acacia catechu suppress HIV-1 replication by inhibiting the activities of the viral protease and tat. Virology J 10: 309, 2013.
33. Dhivya R, Enzo AP, Tiong CY, Diana Lim SL, Chu LT, Francois M and Lara G: Evidence of synergistic activity of medicinal plant extracts against neuraminidase inhibitor resistant strains of influenza viruses. Adv Microbiol 4: 1260-1277, 2014

34. Niazi J, Singh P, Bansal Y and Goel RK: Anti-inflammatory, analgesic and antipyretic activity of aqueous extract of fresh leaves of Coccinia indica. Inflammopharmacology 17: 239-244, 2009.

35. Gopalakrishnan V, Rao KN, Devi M, Padmaha N, Lakshmi PM, Srividya T and Vadivukarasi G: Antihepatotoxic acticity of Coccinia indica. Anc Sci Life 21: 12-15, 2001.

36. Rangu MV, Suresh B, Sandeep VN, Narendra N and Ramesh M: Hepatoprotective activity of leaves of Corallocarpus epigaeus in carbon tetrachloride induced rats. Interl J Biol Pharma Res 3 : 567- 570, 2012

37. Madrigal-Santillán E, Madrigal-Bujaidar E, Álvarez-González I, Sumaya-Martínez MT, Gutiérrez-Salinas J, Bautista M, Morales-González Á, García-Luna y González-Rubio M, Aguilar-Faisal JL and Morales-González JA: Review of natural products with hepatoprotective effects. World J Gastroenterol 20: 14787-14804, 2014

38. Wohlfarth $\mathrm{C}$ and Efferth $\mathrm{T}$ : Natural products as promising drug candidates for the treatment of hepatitis B and C. Acta Pharmacol Sin 30: 25-30, 2009

39. Liu J, McIntosh $\mathrm{H}$ and Lin $\mathrm{H}$ : Chinese medicinal herbs for chronic hepatitis B: A systematic review. Liver 21: 280-286, 2001.

40. Mehrotra R, Rawat S, Kulshreshtha DK, Patnaik GK and Dhawan BN: In vitro studies on the effect of certain natural products against hepatitis B virus. Indian J Med Res 92: 133-138, 1990.

41. Huang RL, Huang YL, Ou JC, Chen CC, Hsu FL and Chang C: Screening of 25 compounds isolated from Phyllanthus species for anti-human hepatitis B virus in vitro. Phytother Res 17: 449-453, 2003.

42. Ott M, Thyagarajan SP and Gupta S: Phyllanthus amarus suppresses hepatitis B virus by interrupting interactions between HBV enhancer I and cellular transcription factors. European J Clin Invest 27: 908-915, 1997.

43. Mehortra R, Rawat S, Kulshreshtha DK, Goyal P, Patnaik GK and Dhawan BN: In vitro effect of Phyllanthus amarus on hepatitis B virus. Indian J Med Res 93: 71-73, 1991.

44. Cheng YC, Ying CX, Leung $\mathrm{CH}$ and Li Y: New targets and inhibitors of $\mathrm{HBV}$ replication to combat drug resistance. J Clin Virol 34 (Suppl 1): S147-S150, 2005

45. Guo Q, Zhao L, You Q, Yang Y, Gu H, Song G, Lu N and Xin J: Anti-hepatitis B virus activity of wogonin in vitro and in vivo. Antiviral Res 74: 16-24, 2007.

46. Li J, Huang H, Feng M, Zhou W, Shi X and Zhou P: In vitro and in vivo anti-hepatitis $B$ virus activities of a plant extract from Geranium carolinianum L. Antiviral Res 79: 114-120, 2008.

47. Jiang Y, Zhang XM, Zhang FX, Liu N, Zhao F, Zhou J and Chen JJ: A new triterpene and anti-hepatitis B virus active compounds from Alisma orientalis. Planta Med 72: 951-954, 2006.

48. Wu YR, Ma YB, Zhao YX, Yao SY, Zhou J, Zhou Y and Chen JJ Two new quaternary alkaloids and anti-hepatitis $b$ virus active constituents from Corydalis saxicola. Planta Medica 73: 787-791, 2007.

49. Chiang LC, Ng LT, Liu LT, Shieh DE and Lin CC: Cytotoxicity and anti-hepatitis B virus activities of saikosaponins from Bupleurum species. Planta Med 69: 705-709, 2003.

50. Li Z, Li LJ, Sun Y and Li J: Identification of natural compounds with anti-hepatitis B virus activity from Rheum palmatum L. ethanol extract. Chemotherapy 53: 320-326, 2007.

51. Huang TJ, Tsai YC, Chiang SY, Wang GJ, Kuo YC, Chang YC, Wu YY and Wu YC: Anti-viral effect of a compound isolated from Liriope platyphylla against hepatitis B virus in vitro. Virus Res 192: 16-24, 2014. 